\title{
Pen-2 overexpression induces $A \beta-42$ production, memory defect, motor activity enhancement and feeding behavior dysfunction in NSE/Pen-2 transgenic mice
}

\author{
SO HEE NAM ${ }^{1 *}$, SU JIN SEO ${ }^{2 *}$, JUN SEO GOO ${ }^{1}$, JEE EUN KIM $^{1}$, SUN IL CHOI ${ }^{1}$, HAE RYUN LEE ${ }^{1}$, \\ IN SIK HWANG ${ }^{1}$, SEUNG WAN JEE ${ }^{2}$, SU HAE LEE ${ }^{2}$, CHANG JUN BAE ${ }^{2}$, JUNG YOUN PARK ${ }^{3}$, \\ HYE SUNG KIM ${ }^{4}$, SUN BO SHIM ${ }^{2}$ and DAE YOUN HWANG ${ }^{1}$
}

\begin{abstract}
${ }^{1}$ Department of Biomaterial Science, College of Natural Resources and Life Science, Pusan National University, Miryang; ${ }^{2}$ Department of Laboratory Animal Resources, National Institute of Food and Drug Evaluation, Korea FDA, Cheongwon; ${ }^{3}$ Biotechnology Research Institute, National Fisheries Research and Development Institute, Busan; ${ }^{4}$ Department of Nanomaterials Engineering, College of Nanoscience and Nanotechnology, Pusan National University, Miryang, Republic of Korea
\end{abstract}

Received June 8, 2011; Accepted July 21, 2011

DOI: 10.3892/ijmm.2011.767

\begin{abstract}
Pen-2 is a key regulator of the $\gamma$-secretase complex, which is involved in the production of the amyloid $\beta(A \beta)-42$ peptides, which ultimately lead to Alzheimer's disease (AD). While Pen-2 has been studied in vitro, Pen-2 function in vivo in the brains of transgenic ( $\mathrm{Tg}$ ) mice overexpressing human Pen-2 (hPen-2) protein has not been studied. This study aimed to determine whether Pen-2 overexpression could regulate the AD-like phenotypes in Tg mice. NSE/hPen-2 Tg mice were produced by the microinjection of the NSE/hPen-2 gene into the pronucleus of fertilized eggs. The expression of the hPen-2 gene under the control of the NSE promoter was successfully detected only in the brain and kidney tissue of NSE/hPen-2 $\mathrm{Tg}$ mice. Also, 12-month-old NSE/hPen-2 Tg mice displayed behavioral dysfunction in the water maze test, motor activity and feeding behavior dysfunction in food intake/water intake/ motor activity monitoring system. In addition, tissue samples displayed dense staining with antibody to the $A \beta-42$ peptide. Furthermore, NSE/hPen-2 Tg mice exhibiting feeding behavior dysfunction were significantly more apt to display symptoms related to diabetes and obesity. These results suggest that Pen-2 overexpression in NSE/hPen-2 Tg mice may induce all
\end{abstract}

Correspondence to: Professor Dae Youn Hwang, Department of Biomaterial Science, College of Natural Resources and Life Science, Pusan National University, 50 Cheonghak-ri, Samnangjin-eup Miryang-si, Gyeongsangnam-do 627-706, Republic of Korea

E-mail: dyhwang@pusan.ac.kr

${ }^{*}$ Contributed equally

Key words: Pen-2, transgenic mice, Alzheimer's disease, feeding behavior, $\mathrm{A} \beta-42$ peptides the AD-like phenotypes, including behavioral deficits, motor activity and feeding behavior dysfunction, $A \beta-42$ peptide deposition and chronic disease induction.

\section{Introduction}

Pen-2 is a component of $\gamma$-secretase, which also consists of a heterodimeric form of presenilins (PSs), a glycosylated mature form of nicastrin (NCT) and APH-1. Pen-2 is responsible for the intramembranous cleavage of $\beta$-amyloid precursor protein (APP) in Alzheimer's disease (AD) (1). Pen-2 was first identified in Caenorhabditis elegans through genetic screening for modifiers of the PS homologues SEL-12 and HOP-1 (2). In humans, Pen-2 is designated PSENEN, and encodes a 101 amino acid polypeptide with a hairpin-like topology, in which both the $\mathrm{N}$ - and C-termini are exposed to the lumen $(3,4)$. Also, Pen-2 is incorporated into the PS-NCT-APH-1 trimeric intermediate during the assembly of the $\gamma$-secretase complex and is responsible for intramembrane proteolysis of a variety of type I membrane proteins including APP, Notch and p75 (5-7). Both the length of the exposed C-terminus and the sequence of a highly-conserved DYLSF Pen-2 motif contribute to the interaction of Pen-2 to other PS complex components in the function of the $\gamma$-secretase during NCT maturation, PS endoproteolysis and the production of amyloid $\beta(\mathrm{A} \beta)$ peptides (8).

Many in vitro studies have reported on the functional role of Pen-2 in the formation and activity of $\gamma$-secretase, although a definitive role has yet to be established. Basically, in most of these studies, Pen-2 overexpression could induce the increase of $\mathrm{A} \beta-42$ production, while the loss of Pen-2 activity resulted in a reduction of PS levels $(2,9,10)$, PS endoproteolysis $(5,6,11)$ and a loss of $\gamma$-secretase activity, which generates $A \beta-42$ peptides, S3-site cleavages and the Notch intracellular domain (NICD) (2). Moreover, cells overexpressing one component of the $\gamma$-secretase complex display a marginal elevation of $\gamma$-secretase activity levels (5). When overexpressed by transient transfection, 
Pen-2 can be incorporated into the endogenous complex with a low degree of efficiency (1). Also, the co-expression of APPsw and Pen-2 can induce minor increases in PS1 fragment levels and a corresponding reduction in the levels of full-length PS1 (5). NCT overexpression induces the increase in $\gamma$-secretase activity without a change in PS component levels (12). However, Pen-2 suppression with transfection of small interfering RNA (siRNA) fragment induces the loss of fragmented forms of PS as well as an accumulation of the PS holoprotein (6), while APH-1 or NCT siRNA do not induce the same result $(2,13)$.

Several reports have described the correlation between Pen-2 and PS with respect to the regulation of gene expression. At the level of transcriptional regulation, Pen-2 mRNA and its promoter transcription were reduced by the depletion of PS. Especially, in fibroblasts this regulation depends on the p53 tumor suppressor (14). Also, at the translational level, PS selectively enhances the stability of Pen-2 and protects it from proteosomal degradation (15). However, there are no studies on the overexpression of human Pen-2 (hPen-2) gene in transgenic ( $\mathrm{Tg}$ ) mice, despite the importance of this approach in investigating the function of Pen-2 and for the screening of AD therapeutic drugs.

The present study addressed this need by developing and characterizing a novel NSE/hPen-2 Tg mouse system. Experiments using these Tg mice implicates Pen-2 in the onset of $\mathrm{AD}$ through the regulation of $\mathrm{A} \beta$ production, behavioral defects and feeding behavior dysfunction.

\section{Materials and methods}

Gene constructions. The $p N S E / h P e n-2$ plasmid, which harbors hPen-2 under the control of the NSE promoter, was previously constructed (16). Briefly, the hPen-2 gene (GenBank accession no. NM-172341) was amplified by polymerase chain reaction (PCR) using a full-length RNA isolated from SK-N-MC cells . The primers used for the amplification were the hPen-2 sense primer, 5'-ggatccATGgaaca aaaac ttatt tctga agatc tg AACCT GGAGC GAGTG TCCAA TG-3' (italics, BamHI site; ATG, start codon; small letters, c-Myc tag; capital letters, hPen-2 sense primer corresponding to nucleotides 2253-2272 of hPen-2) and the hPen-2 antisense primer, 5'-actagt CCAGT TATGT GCAGA AGTTG tca GGG-3' (italics, SpeI site; the capital letters correspond to nucleotides 2253-2272 of hPen-2).

The primers used included an added recognition sequence for the SpeI and XbaI enzymes at the 5' and 3' PCR products, respectively. The amplified $h P e n-2$ was 2,148 bp in length, and the product was cloned into the $\mathrm{T}$ cloning vector $(p G E M-T)$ (Promega, Madison, WI, USA). Sequence analysis was conducted in order to confirm whether or not the cloned $h P e n-2$ sequence was identical to the human Pen-2 $c D N A$, and the resulting sequence was aligned with the NCBI sequence database, using the BLAST program to identify their corresponding hPen-2 gene. The rat NSE (GenBank accession no. AB038993) promoter was also separately PCR-amplified, using pNSE/CAT as a template, which was generously provided by Dr J. GregorSutcliffe at the Research Institute of the Scripps Clinic. The sense primer, 5'-CGTCG ACTAT GGTGG TATGG CTGA-3', harboring nucleotides 37-55, and the antisense primer, 5'-TCGAG GACTG CAGAC TCAG-3', harboring nucleotides 1786-1804, were used. The primers included added recognition sequences for SalI and SpeI to the 5' and 3' PCR products, respectively. The amplified product $(1,777 \mathrm{bp})$ was then cloned into the $p G E M-T$ vector. Following Xhol and EcoRI digestion, the NSE product was inserted into the Xho1 and EcoRI sites of the $r t T A 2 S-M 2$ vector (NSE/rtTA2S-M2). The NSE fragment isolated from NSE/rtTA2S-M2 was cloned upstream of the $c-m y c-h P e n-2$ insert linked to the $S V 40$ polyadenylation signal (pNSE/hPen-2) (Fig. 1A).

Cell cultures and DNA transfection. SK-N-MC human neuroepitheliomal cells were purchased from the Korean Cell Line Bank (Seoul, Korea). Cells were grown in monolayers in minimum essential medium (DMEM; Gibco, Grand Island, NY, USA) supplemented with $10 \%$ fetal bovine serum (FBS; Invitrogen, Carlsbad, CA, USA) and antibiotics (100 U/ml penicillin and $100 \mu \mathrm{g} / \mathrm{ml}$ streptomycin; Gibco) during incubation at $37^{\circ} \mathrm{C}$ in a humidified incubator containing $5 \% \mathrm{CO}_{2}$ in air. All other chemicals were purchased from Sigma-Aldrich (St. Louis, MO, USA).

To overexpress the Pen-2 protein in neuroblastoma cells, transfection of NSE/hPen-2 plasmid was performed using the Lipofectamine $^{\mathrm{TM}}$ reagent (Invitrogen). SK-N-MC cells were seeded at a density of $2 \times 10^{6}$ cells per $100 \mathrm{~mm}$-diameter dish. After reaching 80-90\% confluence, a plasmid-lipofectamine mixture containing $10 \mu \mathrm{g}$ of DNA (per dish) was added, and the cells were incubated in OptiMEM medium for an additional $5 \mathrm{~h}$ at $37^{\circ} \mathrm{C}$. Then, the cells were cultured in the fresh serum-free medium for $24 \mathrm{~h}$ and were used for the Western blot analysis and flow cytometry analyses subsequently described.

Flow cytometry analysis. The expression of the Pen-2 gene in SK-N-MC cells transfected with NSE/Pen-2 plasmid was detected by flow cytometry after staining with a Pen-2 specific antibody. After an additional $24 \mathrm{~h}$ incubation after transfection, all cells were harvested, washed twice in ice-cold phosphate buffered saline (PBS), and resuspended in $1 \mathrm{X}$ binding buffer at a concentration of $1 \times 10^{6}$ cells $/ \mathrm{ml}$. Approximately $1 \times 10^{5}$ cells in $100 \mu \mathrm{l}$ of solution were transferred to round-bottomed culture tubes. Five microliters of fluorescein isothiocyanate (FITC)conjugated anti-Pen-2 antibody was added to stain the cells. After 15 min of incubation at room temperature, $400 \mu 1$ of $1 \mathrm{X}$ binding buffer was added to each tube and each sample was analyzed with a FACScalibur apparatus (BD Biosciences, Franklin Lakes, NJ, USA) within 1-2 h.

Care and use of animals. All animal experimental procedures were approved by the Institutional Animal Care and Use Committee (IACUC) at the Korea Food and Drug Administration (KFDA) and the Pusan National University. All mice were supplied by the breeding center of the Korea FDA facility and were handled in a PNU-Laboratory Animal Resources Center and a Korea FDA-accredited animal facility in accordance with the AAALAC International Animal Care policy (Accredited Unit, Korea Food and Drug Administration, unit no. 000996). Mice were housed in cages under a strict light cycle (lights on at 06:00 $\mathrm{h}$ and off at 18:00 h) and temperature $\left(23 \pm 1^{\circ} \mathrm{C}\right)$. In addition, all mice were provided with a standard irradiated chow diet (Purina Mills, St. Louis, MO, USA) ad libitum and maintained in a specific pathogen free (SPF) state. 
Production of NSE/hPen-2 Tg mice. The $p N S E / h P e n-2$ plasmid was digested with $B g l I \mathrm{II} / P v u I I$ in order to remove the prokaryotic sequence and electrophoresed on an agarose gel. The linear gene fragment of the $p N S E / h P e n-2$ fragment (2,254-bp) was excised and extracted from the agarose gel. The $p N S E / h P e n-2$ fragment was purified by electropurification, diluted to $4 \mathrm{ng} / \mu 1$ and microinjected into the male pronucleus of fertilized embryos, which were obtained by the crossing a female with a male BDF1 mouse originated from a mating between female C57BL/6 and male DBA/2 mice (17). The injected egg was then transferred into the oviducts of a pseudopregnant ICR recipient female on Day 1. The founder mice, into which the NSE/hPen-2 transgene had been inserted, were identified by DNA-PCR of the tailderived DNA. For DNA-PCR, 10 pmole of the sense, 5'-GCT ATG AAC CTG GAG CGA GTG-3' and antisense, 5'-GAA GGA GAG GTA GTC CCC AAG G-3' primers were added into genomic DNA template mixture, and the reaction mixtures were subjected to 25 cycles of amplification. Amplification was conducted in a thermal cycler (Perkin-Elmer, Waltham, MA, USA), under the following conditions: $30 \mathrm{sec}$ at $94^{\circ} \mathrm{C}, 30 \mathrm{sec}$ at $62^{\circ} \mathrm{C}$ and $45 \mathrm{sec}$ at $72^{\circ} \mathrm{C}$. The amplified PCR products were separated on a $1 \%$ agarose gel and the bands were detected using the Kodak Electrophoresis Documentation and Analysis System 120 (Eastman Kodak, Rochester, NY, USA). The Tg founder mice were then crossed onto the parental strain of the C57/BL6 background to establish the transgenic lines. Subsequently, all pedigrees were hemizygous for their transgene.

Reverse transcription (RT)-PCR analysis. For the preparation of total-RNA, tissues frozen in liquid nitrogen were chopped with scissors and homogenized in RNAzol B solution (Tet-Test, Austin, TX, USA). The isolated RNA was then quantified using an Ultraspec 1000 system (Amersham Pharmacia Biotech, Buckinghamshire, UK). To characterize the expression of transgenes, RT-PCR was conducted using $5 \mu \mathrm{g}$ of total-RNA from each of the tissue samples. Oligo(dt) primers of $500 \mathrm{ng}$ (Gibco-BRL, Grand Island, NY, USA) were annealed for $10 \mathrm{~min}$ at $70^{\circ} \mathrm{C}$. Complementary DNA, which was utilized as a template for further amplification, was synthesized via the addition of dATP, dCTP, dGTP and dTTP, as well as 200 units of reverse transcriptase. Thereafter, 10 pmole of the sense and antisense primers were added, and the reaction mixtures were subjected to 28 cycles of amplification. Amplification was conducted in the afore-mentioned thermal cycler under the afore-mentioned conditions. In each case, minus-RT controls were included to distinguish between the DNA and RNA products. This experiment was repeated three times, and the relative differences in RNA quantity were also reproducibly observed in the three experiments. Finally, the levels of the Pen-2 RT-PCR product were quantified using the afore-mentioned electrophoresis documentation and analysis system on a $1 \%$ agarose gel.

Western blotting and slot blotting. For slot blotting, protein prepared from cells transfected with NSE/SV4O and NSE/ hPen-2 plasmids were transferred to a nitrocellulose membrane using a Slot Blot kit (Amersham Pharmacia Biotech). The membrane was incubated separately with primary rabbit polyclonal anti-A $\beta-42$ unconjugated at $2 \mu \mathrm{g}$ in blocking buffer at room temperature for $3 \mathrm{~h}$, and were washed in washing buffer and then incubated with secondary antibody, alkaline phosphatase-conjugated goat anti-rabbit $\operatorname{IgG}$ at $1: 1,000$ for $1 \mathrm{~h}$ at room temperature. The slot band for the $A \beta-42$-specific peptide was detected by an NBT/BCIP substrate. For Western blotting, $10 \mu \mathrm{g}$ of protein were separated by electrophoresis on a $10 \%$ polyacrylamide gel for $3 \mathrm{~h}$ and the resolved species were transferred to a nitrocellulose membrane by electroblotting for $2 \mathrm{~h}$. The membrane was incubated with the following primary antibodies: anti-human Pen-2 (Calbiochem, San Diego, CA; 1:1,000 dilution), anti-Glut-1 (Abcam, Cambridge, UK; 1:1,000 dilution), anti-Glut-3 (Abcam; 1:1,000 dilution) or anti-actin (Sigma-Aldrich; 1:1,000 dilution) overnight at $4^{\circ} \mathrm{C}$. Each membrane was washed with buffer $(137 \mathrm{mM} \mathrm{NaCl}$, $2.7 \mathrm{mM} \mathrm{KCl}, 10 \mathrm{mM} \mathrm{NaHPO}$ and $0.05 \%$ Tween-20) and incubated with a 1:1,000 dilution of horseradish peroxidase (HRP)-conjugated goat anti-rabbit IgG at room temperature for $2 \mathrm{~h}$. The membrane blots were developed using the Enhanced Chemiluminescence Reagent Plus kit (Amersham Biosciences).

Perfusion and immunohistochemical analysis. Brain perfusion and immunohistochemical analysis was performed as previously described $(18,19)$. Briefly, mice were anesthetised with Zoletil 50 (Virbac, Carros cedex, France) and transcardially perfused with $1 \mathrm{X}$ PBS followed by buffered $4 \%$ formaldehyde to effectively remove the blood and fix the brain tissue. After perfusion, each mouse brain was isolated from the skull and fixed overnight in formaldehyde. Each brain was dehydrated and embedded in paraffin. A series of brain sections $(10 \mu \mathrm{m})$ were cut from paraffin-embedded tissue using a Leica microtome (Leica Microsystems, Bannockbrun, IL, USA). For immunohistochemical analysis, these sections were de-paraffinized with xylene, rehydrated and pretreated for $30 \mathrm{~min}$ at room temperature with PBS blocking buffer containing 10\% goat serum. Next, the sections were incubated with mouse anti-Pen-2 antibody and anti-A $\beta-42$ antibody (Invitrogen), both at a dilution of 1:100 in PBS blocking buffer. The antigen-antibody complexes were visualized with biotinylated secondary antibody (goat anti-rabbit)-conjugated HRP streptavidin (Histostain-Plus kit; Zymed, South San Francisco, CA, USA), at a dilution of 1:1,500 in PBS blocking buffer. Pen-2 protein and $A \beta-42$ peptide were detected using stable 3,3'-diaminobenzidine (DAB; Invitrogen) and observed using the BX50F-3 optical microscope (Olympus, Tokyo, Japan).

Water maze test. Twelve-month-old mice were subjected to water maze tests following a previously-described procedure (18). Briefly, the tests were performed using the SMART-CS (Panlab, Barcelona, Spain) program, which was placed in an experimental room with a window, air-conditioning and tables. This experiment was conducted in a plastic, $1.5 \mathrm{~m}$-diameter circular pool filled with water maintained at $20-22^{\circ} \mathrm{C}$. The visual field of the water was obstructed by the addition of powdered milk. Mice were pre-trained by allowing them to swim to a round platform (diameter $12 \mathrm{~cm}$ ) submerged $1 \mathrm{~cm}$ beneath the surface. The escape latencies, escape distances, swimming speeds and swimming patterns of the mice were monitored by the SMART-LD computer program, which was connected to a camera mounted to the ceiling directly above the pool. Prior to the experiment, a $60 \mathrm{sec}$ habituation trial 
was performed to verify that the mice could swim. The mice were then given five training trials in which their ability to find the hidden platform was measured for a maximum of $60 \mathrm{sec}$. If the mice failed to find the platform within the maximum time, they were physically placed on it. The training schedule consisted of two trials per day over 5 test days, and each trial was assessed based on the ability of the mouse to reach the platform within $60 \mathrm{sec}$. The second trial was conducted at least $5 \mathrm{~min}$ after the first one. However, the two trials were started from identical locations and the platform location was kept constant during the training period. After each trial, the mice were allowed to remain on the platform for $30 \mathrm{sec}$. On the sixth day, the mice were subjected to three probe trials, during which they swam for $60 \mathrm{sec}$ with no platform in the pool. In this test, each of the two training trials and three actual trials were initially started from the right side of the water pool, and then from the opposite side. The patterns of searching, the number of times the mice swam to the former location of the platform (escape latency), the distances the mice swam (escape distance) and the swimming speeds (velocity) to the former location of the platform were recorded. All trials were recorded and stored on a videotape for subsequent analysis.

Feeding behavior and motor activity analysis. Feeding behavior and motor activity of 12-month-old NSE/hPen-2 $\mathrm{Tg}$ mice were detected by a food intake/water intake/motor activity monitoring system MDF-100 (Shinfactory, Tokyo, Japan). One NSE/hPen-2 Tg mouse and one non-Tg mouse that had been housed in groups (4-6 mice/cage) until 12-monthsof-age and had originated from same parent were taken and separated into individual chambers of the monitoring system. Food intake, water intake and motor activity were monitored for at least 2 weeks. The data of three parameters were collected from 3-5 mice/group using ACTIMO-S Vista-compliant data acquisition software (Shinfactory, Tokyo, Japan).

Serum biochemical analysis. Following the final application of feeding behavior and motor activity, the mice were fasted for $24 \mathrm{~h}$ and whole blood was collected from their abdominal vein. Serum was obtained by centrifuging the blood $\left(15,000 \mathrm{rpm}, 4^{\circ} \mathrm{C}, 10 \mathrm{~min}\right)$, followed by incubation for $30 \mathrm{~min}$ at room temperature. The serum was then stored at $-80^{\circ} \mathrm{C}$ until analysis. The glucose, total serum cholesterol and triglyceride were assayed in duplicate on fresh serum using a model 747 automatic serum analyzer (Hitachi, Tokyo, Japan).

A $\beta$-42 enzyme-linked immunosorbent assay (ELISA). A $\beta-42$ levels in the brain of NSE/hPen-2 Tg mice were measured as previously described (20). Firstly, the cortex region was collected from the brain of non-Tg and NES/hPen-2 Tg mice, and homogenized in Tris-buffered saline (20 mM Tris, $137 \mathrm{mM} \mathrm{NaCl}, \mathrm{pH}$ 7.6). The supernatant containing soluble $\mathrm{A} \beta-42$ was prepared from the homogenizer mixture using centrifugation at $100,000 \times \mathrm{g}$ for $1 \mathrm{~h}$. The levels of $\mathrm{A} \beta-42$ peptides were analyzed using Signal Select Human $\beta$ Amyloid (1-42) colorimetric sandwich ELISA kits (Signal Select ${ }^{\mathrm{TM}}$, BioSource, Camarillo, CA, USA) following the manufacturer's instructions. The standard, control and brain samples were added into $A \beta-42$ antibody-coated wells of 96-well plates, and $50 \mu \mathrm{l}$ of detection antibody solution were immediately added. After the incubation for $3 \mathrm{~h}$, the unbound proteins were removed with washing steps and $100 \mu \mathrm{l}$ of anti-rabbit IgG-HRP working solution was immediately added to the plate. Finally, the level of A $\beta-42$ peptides were measured to read the absorbance of color formed by the reaction between the stabilized chromogen and the stop solution at $450 \mathrm{~nm}$.

Insulin ELISA. The insulin levels in serum obtained from non-Tg and NSE/hPen-2 Tg mice were detected using the ultra-sensitive assay procedure and reagents in the insulin ELISA kit (Mercodia, Uppsala, Sweden). Briefly, serum and standards were incubated in a plate shaker at $100-150 \mathrm{rpm}$ at room temperature for $2 \mathrm{~h}$ on antibody-coated plates. The wells were then washed six times with an automated plate washer (Hoefer, Holliston, MA, USA), after which horseradish peroxidase (HRP) conjugate was added to each of the wells. The plates were then incubated in a shaker for $30 \mathrm{~min}$ at room temperature. The reaction was terminated by the addition of $50 \mu 1$ of stop solution $\left(0.5 \mathrm{M} \mathrm{H}_{2} \mathrm{SO}_{4}\right)$, after which the plates were analyzed by evaluating the absorbance at $450 \mathrm{~nm}$ using a Molecular Devices $\mathrm{V}_{\max }$ Plate reader (Sunnyvale, CA, USA).

Statistical analysis. Tests for significance were conducted using one-way ANOVA analyses of variance (SPSS for Windows, release 10.01; SPSS, USA). All values were expressed as the mean \pm standard deviation (SD). Significance was set as a value of $\mathrm{P}<0.05$.

\section{Results}

Identification of hPen- 2 protein expression and $A \beta-42$ production in neuroblastoma cells. Prior to testing of the effects of hPen-2 overexpression in Tg mice, the NSE/hPen-2 plasmid was applied to detect the expression of hPen- 2 protein in neuroblastoma cells and quantify the $A \beta-42$ levels produced by $\gamma$-secretase activation. After transfection with NSE/hPen-2 or NSE/SV4O plasmid, the $12-\mathrm{kDa}$ Pen-2 protein was detected by Western blotting using a Pen- 2 specific antibody. The levels of Pen-2 protein were determined to be elevated only in cells transfected with NSE/hPen-2 as compared to those transfected with NSE/SV4O (Fig. 1Ba). Also, fluorescence activated cell sorting (FACS) analysis revealed that Pen-2 proteins in the cell membrane were significantly increased and successfully detected in cells transfected with NSE/hPen-2 (Fig. 1Bb). Furthermore, the level of $\mathrm{A} \beta-42$ peptides was higher in NSE/ hPen-2 transfectants than in NSE/SV4O transfectants (Fig. 1C). The results from transiently-transfected cells suggest that the NSE/hPEN-2 plasmid may be useful for this study, as it mimics conditions of the actual in vivo abnormally enhanced Pen-2 overexpression.

Identification of NSE/hPen-2 Tg mice. To produce NSE/ hPen-2 Tg mice overexpressing the hPen-2 protein in each tissue, the 2,254-bp NSE/hPen-2 fragment was microinjected into the male pronucleus of fertilized embryos obtained by crossing a female with a male BDF1 mouse. Of a total of 118 offspring, eight mice (\#6, 18, 21, 46, 49, 60, 86 and 95) from the first lineage founder mice possessed the NSE/hPen-2 gene, which was identified by genomic DNA-PCR using the hPen-2 specific primer (Fig. 1D). The founder mice containing the 
A
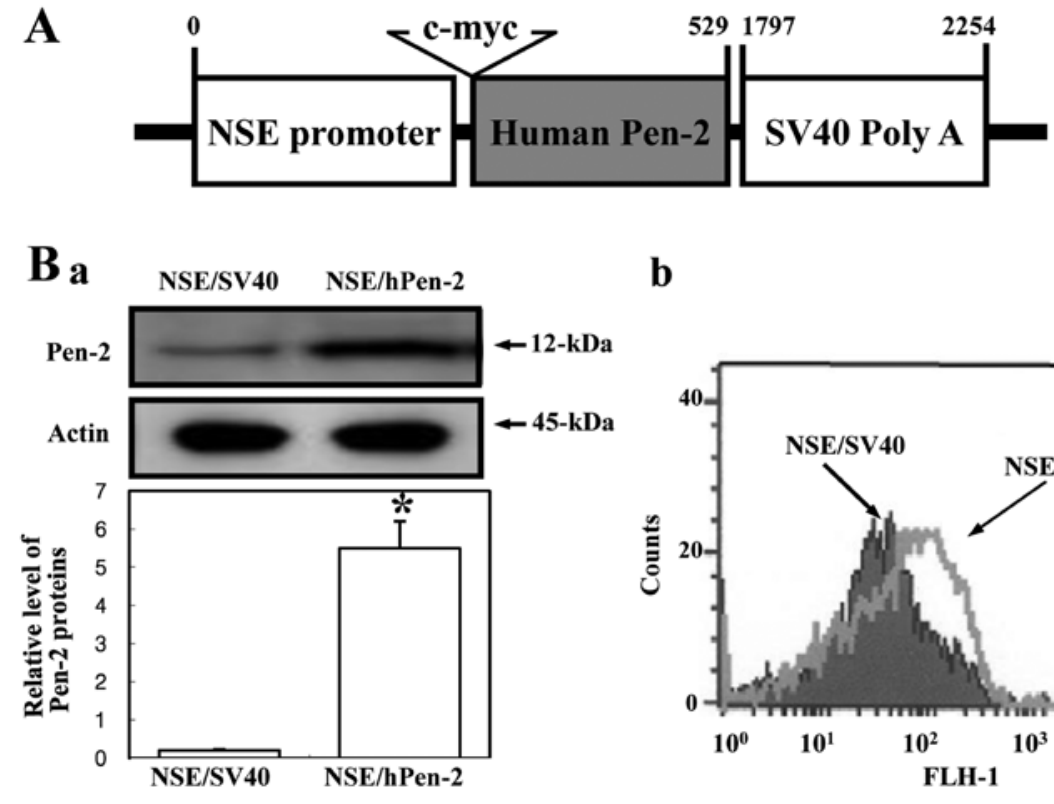

b

$\mathbf{C a}_{\mathbf{a}}$
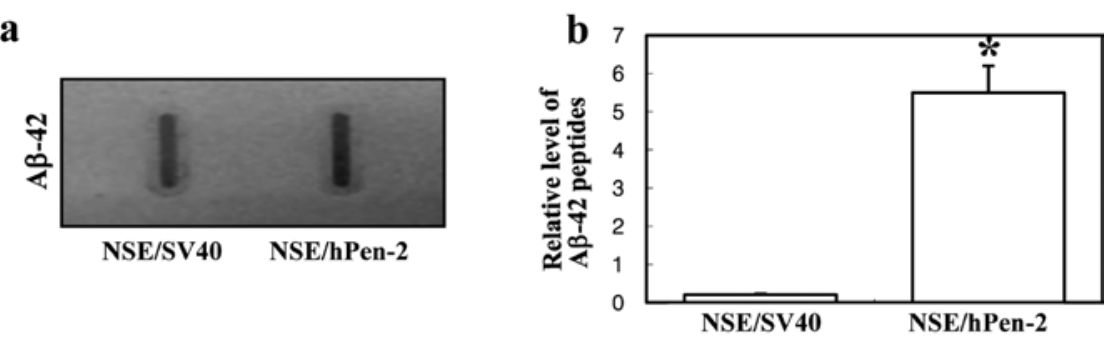

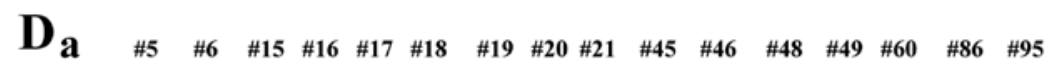

\begin{tabular}{|c|c|c|c|c|}
\hline b & Gene & $\begin{array}{l}\text { Number of offspring } \\
\text { (mice) }\end{array}$ & $\begin{array}{l}\text { Number of } \mathbf{T g} \\
\quad \text { (mice) }\end{array}$ & $\begin{array}{l}\text { Efficiency } \\
(\%)\end{array}$ \\
\hline & NSE/hPen-2 & 118 & 8 & 6.77 \\
\hline
\end{tabular}

Figure 1. The construction of NSE/hPen-2, the expression of Pen-2 protein in neuroblastoma cells and identification of the Tg mice using genomic DNA-PCR. (A) The construction of $p N S E / h P e n-2$. pNSE/hPen-2 harbors the encoding $h P e n-2$ gene under the control of the NSE gene promoter. (Ba) Confirmation of the expression of the Pen-2 protein. Cells were transiently transfected with $p N S E / h P e n-2$, and their expression level was detected by Western blotting. The bands were quantified to obtain the relative protein levels, and the value was defined as 1 (NSE/SV40) to assess other relative values. (Bb) Confirmation of the corrective formation and location of the Pen-2 protein using FACS analysis. After transfection, the neuoblastoma cells were stained with FITC-conjugated anti-Pen-2 antibody. The fluorescence emitted from these cells was analyzed using flow cytometry. Dark gray indicates the intensity in the cells transfected with the NSE/ $S V 40$, and light gray indicates the intensity in the cells transfected with the NSE/hPen-2. (C) Detection of A $\beta-42$ levels using slot blotting analysis. Total soluble extract of transfected cells was applied onto a Slot Blot kit to bind $\mathrm{NC}$ membrane and A $\beta-42$ levels were measured with a specific antibody as described in Materials and methods. (Da) PCR was performed on the genomic DNA isolated from the tail of founder mice, and the resulting products (284 bp) are shown. (Db) Efficiency of Tg mice production. The values are expressed as the mean $\pm \mathrm{SD}$ of three experiments. " $\mathrm{P}<0.05$ vs. cells transfected with $N S E / S V 40$.

NSE/hPen-2 gene were then mated with the C57BL/6 mice to produce a large number of animals. The NSE/hPen-2 gene introduced into their genomes was transmitted to all offspring with approximately $50 \%$ hemizygotes in a Mendelian fashion.

Tissue-specific regulation of hPen-2 expression in the NSE/ hPen-2 Tg mice. To determine whether the regulation of the introduced $h P e n-2$ gene was expressed under the control of the NSE promoter in neural tissues, the transcriptional levels of Pen-2 from various tissues, including the brain, heart, lung, liver, kidney, intestine and muscles of $\mathrm{Tg}$ mice were examined by RT-PCR analysis. The RT-PCR analysis showed that the highest level of hPen-2 expression was observed in the kidney, followed by the brain and heart (Fig. 2A). Furthermore, to detect the localization and distribution of the Pen-2 protein in brain tissues, Pen-2 protein immunoreactivity was analyzed in the brain using optical microscopy. The immunostaining intensity in the NSE/hPen-2 Tg mice was spread throughout the CA1-3 of the hippocampus and dentate gyrus (DG) in the brain (Fig. 2B). However, the level of intensity in the non-Tg 
A

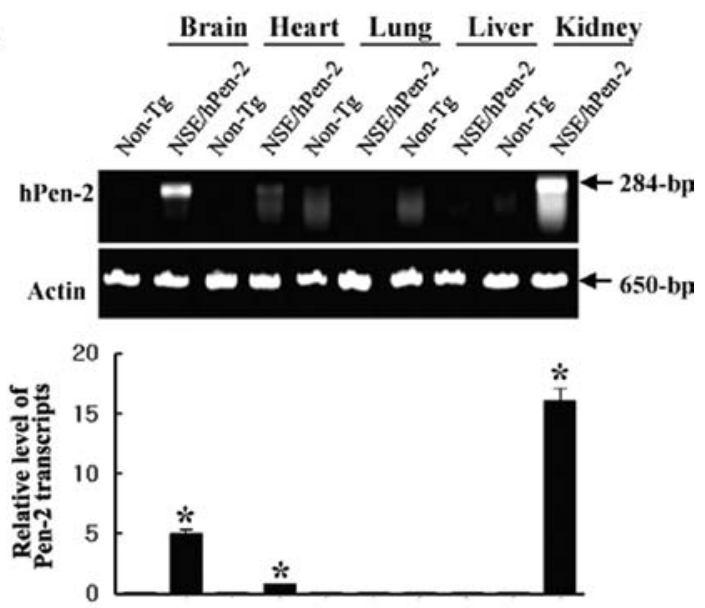

B

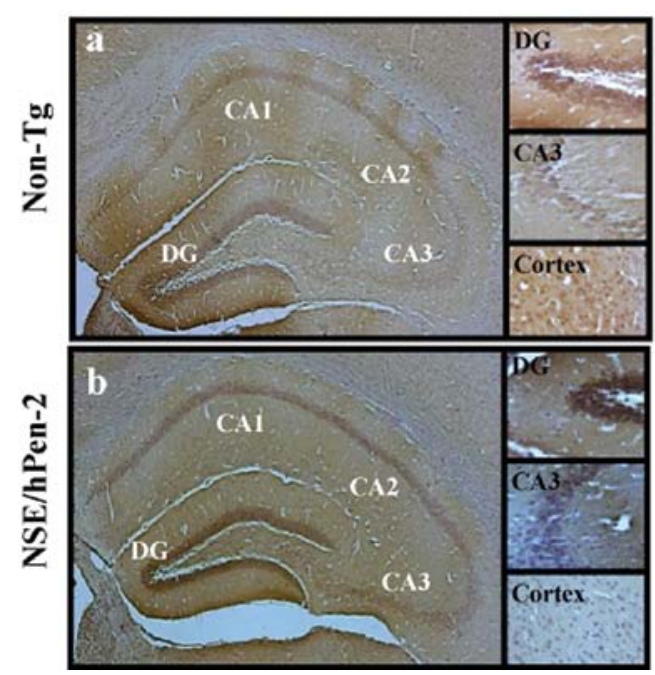

Figure 2. Tissue-specific expression of the hPen-2 transgenes in the $\mathrm{Tg}$ mice. (A) Expression of $h P e n-2$ in various tissues by RT-PCR. The $\beta$-actin signal was used as the control, and the transcript $(650 \mathrm{bp})$ indicates the RNA loading. In addition, the RT-PCR products for $\mathrm{hPen}-2$ (284 bp) are indicated. The density of the transcript was quantified. (B) Immunostaining analysis of Pen-2 expression. The expression profile of Pen-2 in the brain of 12-months of age animals was detected by immunostaining analysis. A high intensity was observed in the hippocampus (CA1-3) and dentate gyrus (DG) of the NSE/hPen-2 Tg mice (Ba) as compared with the non-Tg mice (Bb) at x200 magnification. Also, the detailed histological features of several regions in the hippocampus are shown in the three rectangles on the right at $x 400$ magnification. The data represent the mean $\pm \mathrm{SD}$ from three replicates. ${ }^{*} \mathrm{P}<0.05$; significant difference between NSE/hPen-2 Tg and non-Tg mice.

littermates was slightly lower than that of the NSE/hPen-2 Tg mice (Fig. 2B). Therefore, all of the above observations indicate that the regulatory sequence of the NSE promoter could be successfully attributed to the synthesis of the hPen-2 transcripts and protein in the NSE/hPen-2 Tg mice.

Behavioral deficits of NSE/hPen-2 Tg mice. To assay for early behavioral defects, the escape latency and distance, and swimming patterns and speeds were measured in 12-month-old non-Tg and NSE/hPen-2 Tg mice by water maze tests. After the last training (Day 5), all the mice were given three prove trials on Day 6, in which they swam in the pool for $60 \mathrm{sec}$ with the platform removed. NSE/hPen-2 Tg mice appeared to be slightly impaired, about $30 \%$ than the non-Tg mice, in both the escape latencies (Fig. 3A) and distances (Fig. 3B and D).
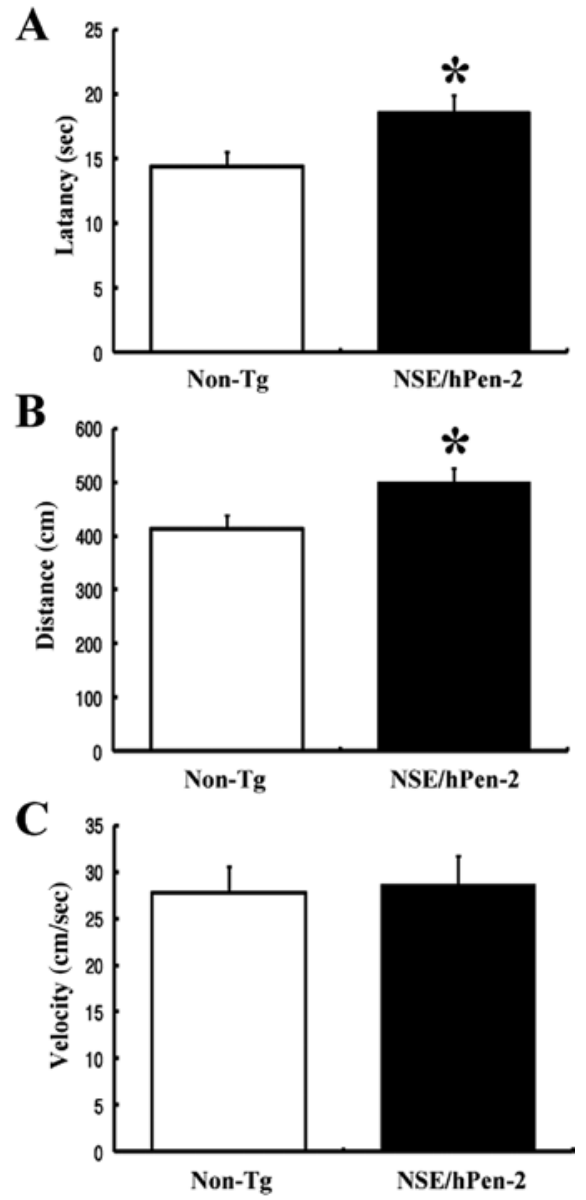

D

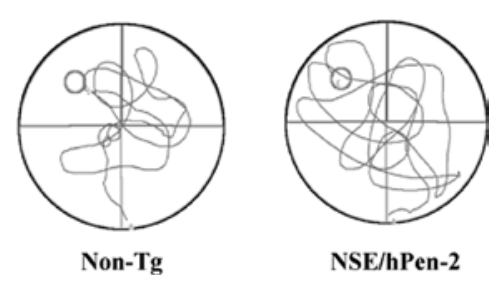

Figure 3. Water maze tests in 12-month-old NSE/hPen-2 Tg mice. Patterns of the (A) escape latency, (B) escape distance and (C) swimming velocity to cross to the former platform location in the water maze. In this test, the data from the training (5 days) and prove trials (1 day) were indicated. The NSE/ hPen-2 Tg groups showed a consistent trend toward longer escape latencies and longer distances than those in the non-Tg group. There were no significant differences in the swimming velocities between the groups. The patterns of the escape latency in the swimming pool are indicated for the 2 groups (D). The results are reported as the median \pm SD of triplicate experiments performed with the water maze $(n=5)$. " $\mathrm{P}<0.05$; significant differences between NSE/hPen-2 Tg and non-Tg mice.

However, the speed of swimming (velocity) did not differ between non-Tg and NSE/hPen-2 Tg mice (Fig. 3C). Thus, these results show that overexpression of the $h P e n-2$ gene in the brain of the NSE/hPen-2 Tg mice led to an accelerated brain deficit on learning and memory.

Alterations of motor activity in NSE/hPen-2 Tg mice. The motor activity was assessed by measurement of infrared-beam breaks in a food intake/water intake/motor activity monitoring system (21). Motor activity of NSE/hPen-2 Tg mice was significantly increased compared to non-Tg mice (Fig. 4A). This hyperactivity was predominant in the dark phase for $12 \mathrm{~h}$ (Fig. 4B). Especially, 
A

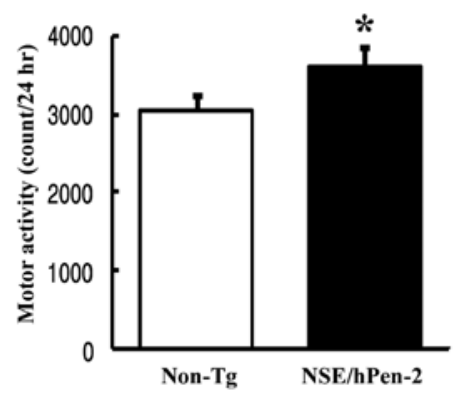

C a

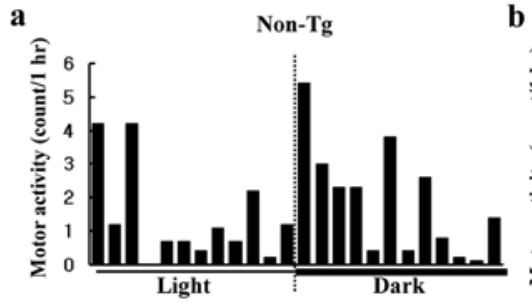

B

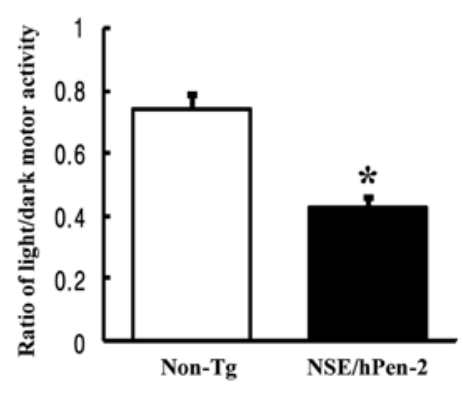

b

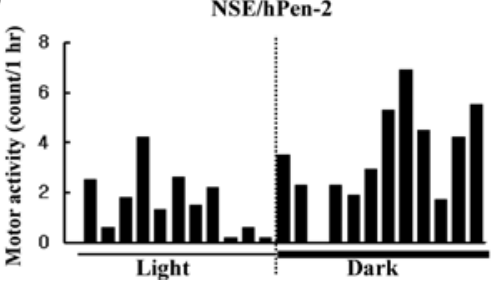

Figure 4. Alteration of the motor activity of NSE/hPen-2 Tg mice. (A) Total motor activity for $24 \mathrm{~h}$. (B) Ratio of light to dark motor activity. (C) Spontaneous locomotor activity of NSE/hPen-2 Tg mice at 12 -months of age expressed as the count of infrared-beam splits during the light and dark phases. * $\mathrm{P}<0.05$; significant differences between NSE/hPen-2 Tg and non-Tg mice.

A $\mathbf{a}$

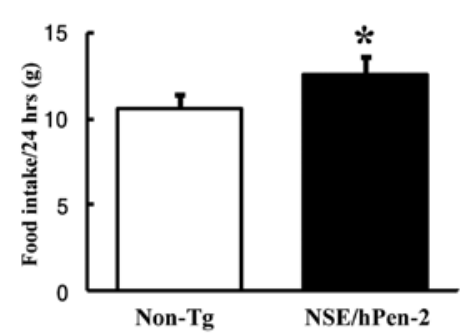

b

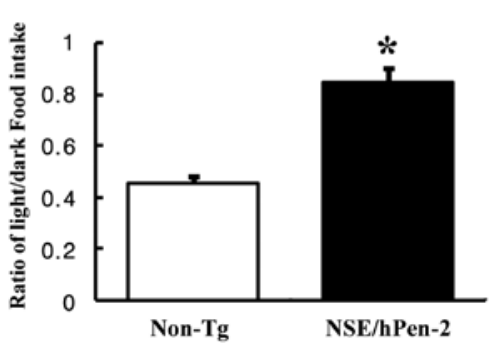

c

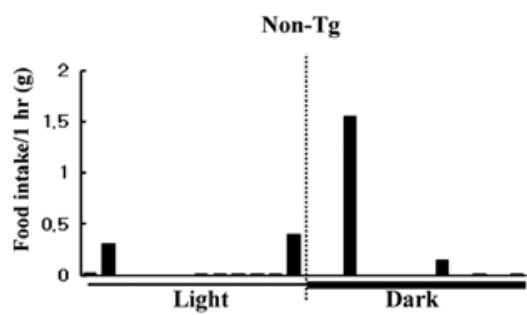

c'

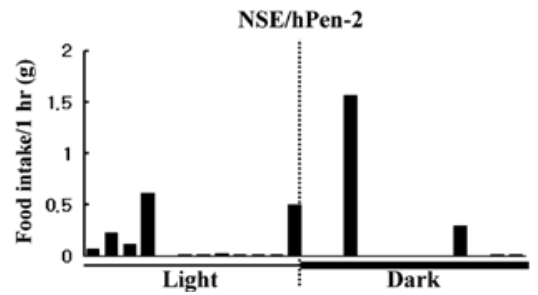

B $\mathbf{a}$

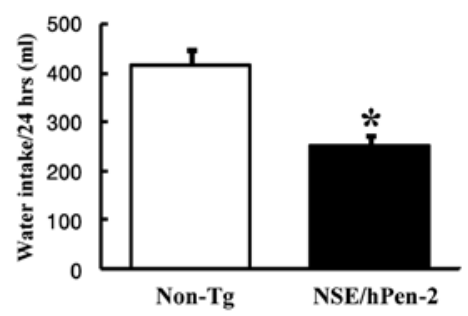

b

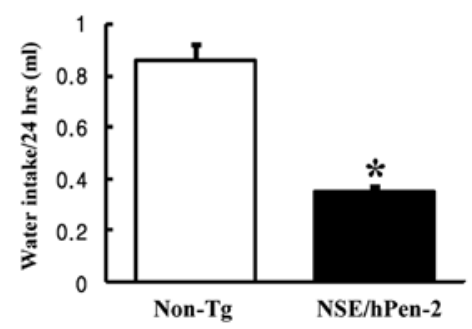

c

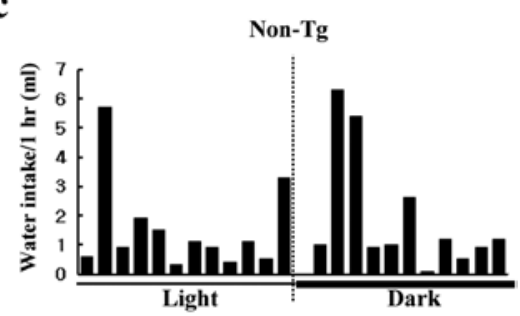

c'

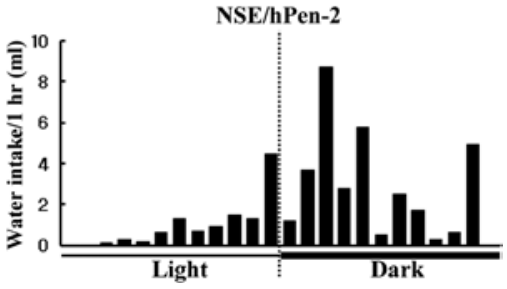

Figure 5. Alteration of (A) daily food and (B) water intake of NSE/hPen-2 Tg mice. Each column expresses (Aa and Ba) the total amount of feeding for 24 h, and $(\mathrm{Ab}$ and $\mathrm{Bb})$ the ratio of light/dark on the amount of feeding. Each bar in (Ac and c' and Bc and c') indicate the amount of food intake and water intake for $1 \mathrm{~h}$ of the light phase or dark phase. ${ }^{*} \mathrm{P}<0.05$; significant differences between NSE/hPen-2 Tg and non-Tg mice. 

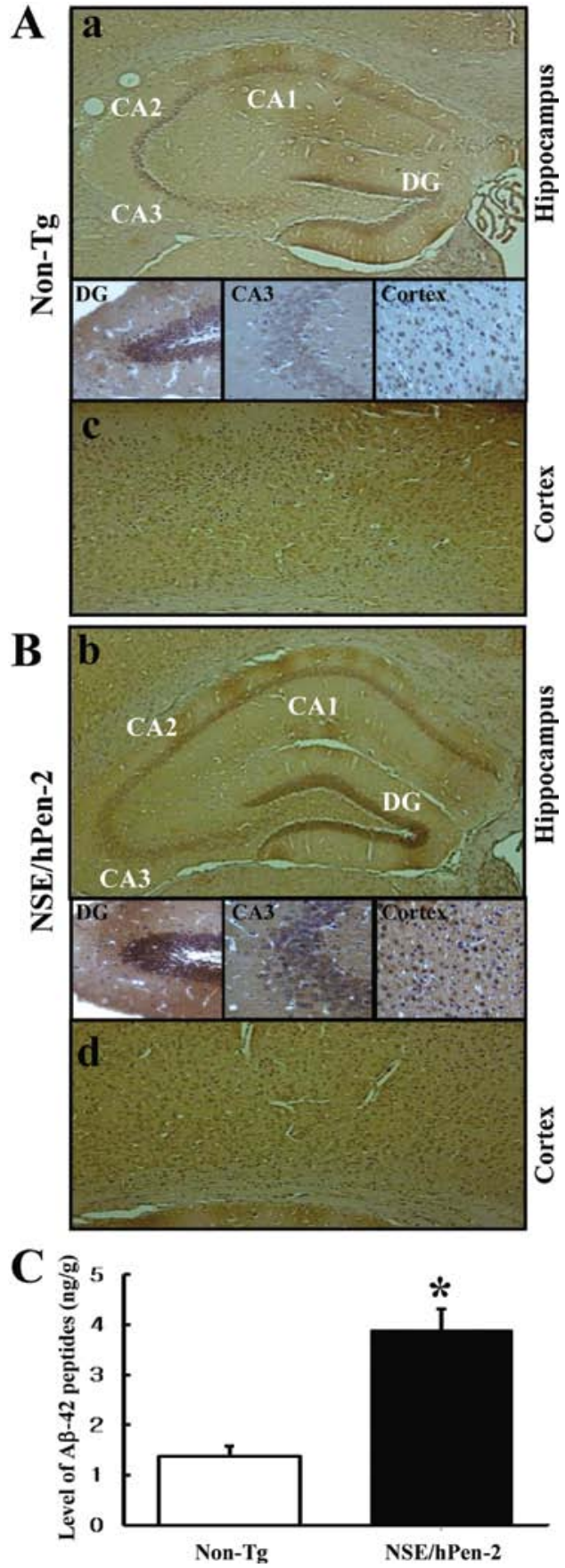

Figure 6. Immunostaining analysis and ELISA of $A \beta-42$ peptide. (A) The brains were taken from NSE/hPen-2 Tg mice and non-Tg mice after perfusion. The level of $A \beta-42$ peptide was detected by immunostaining analysis using an $\mathrm{A} \beta$-42-specific antibody. A high intensity was observed in the hippocampus (CA1-3) and dentate gyrus (DG) of the NSE/hPen-2 Tg mice (Bb) as compared with the non-Tg mice (Aa) at x200 magnification. Also, the highly dense cells were distributed in the cortex region of NSE/hPen-2 Tg mice (Bd) compare with non-Tg mice (Ac). (C) Quantification of A $\beta-42$ level. Level of $A \beta-42$ peptides was measured by sandwich ELISA as described in Materials and methods. Three experiments were assayed in triplicate using ELISA. The values represent the mean $\pm \mathrm{SD}$. ${ }^{*} \mathrm{P}<0.05$; significant difference between NSE/hPen-2 Tg and non-Tg mice.

in the late stage of the dark phase, the motor activity of NSE/ hPen-2 Tg mice was higher, while non-Tg mice showed higher motor activity in the early phase of the dark phase (Fig. 4C). These results suggest that Pen-2 overexpression in NSE/hPen-2 $\mathrm{Tg}$ mice could contribute to the hyperactivity in the dark phase.
Alterations of the feeding behavior of NSE/hPen-2 Tg mice. Changes in the eating behavior or diet are common in dementia such as frontotemporal dementia, AD and Parkinson's disease $(22,23)$. To evaluate and characterize the difference in the feeding behavior between non-Tg and NSE/hPen-2 Tg mice, the amount of food and water consumption were detected by the food intake/water intake/motor activity monitoring system. The feeding behavior of NSE/hPen-2 Tg mice differed markedly from that of non-Tg mice. Daily food consumption was significantly greater in NSE/hPen-2 Tg mice than non-Tg mice (Fig. 5Aa). The ratio of food consumption in the light versus dark phase was dramatically increased in NSE/hPen-2 Tg mice compared with non-Tg mice (Fig. 5Ab and c). Especially, NSE/ hPen-2 Tg mice took lots of pellets at the early stage of light phase (Fig. 5Ac'). However, the water intake by NSE/hPen-2 Tg mice was decreased by $50 \%$ compared with the water intake of non-Tg mice (Fig. 5Ba). These decreases of water consumption were mainly observed at the late stage of the light phase than the dark phase (Fig. 5Ab, c and c'). These results showed that Pen-2 overexpression of NSE/hPen-2 Tg in 12-month-old mice may induce changes in eating and drinking behavior.

A $\beta-42$ peptide deposition in the brain of NSE/hPen-2 Tg mice. To determine whether hPen-2 overexpression in NSE/hPen-2 $\mathrm{Tg}$ mice is accompanied by elevated levels of $\mathrm{A} \beta-42$ peptides produced by $\gamma$-secretase including APH-1, NCT and full length PS2 (PS2-FL) as well as the PS2 fragment (PS2-CTF), immunostaining analysis was conducted for the hippocampus region of the brain using an $\mathrm{A} \beta-42$ peptide-specific antibody. Levels of $A \beta-42$ peptide were higher in the CA1-3 of hippocampus (Fig. 6Bb), DG (Fig. 6Bb) and cortex region (Fig. 6Bd) of NSE/hPen-2 Tg mice than in those of non-Tg mice (Fig. 6Aa and c). Also, a significant increase of $A \beta-42$ peptides in soluble mixture of NSE/hPen-2 Tg mice brain was detected by ELISA (Fig. 6C). These results showed that Pen-2 protein overexpression in the brain of NSE/hPen-2 Tg mice may induce the production and deposition of $A \beta-42$ peptide.

Effect of feeding behavior dysfunction induced by Pen-2 overexpression on diabetes and obesity. In humans, the alteration of feeding behavior is considered to be the major cause for diseases such as diabetes, obesity, cardiovascular disease and hypertension (24). To explore whether the feeding behavior dysfunction could induce the alteration of an indicator for diabetes and obesity, the level of some parameters including glucose, insulin, cholesterol and triglyceride were measured in the serum of NSE/hPen-2 Tg mice. As shown in Fig. 7A and B, cholesterol and triglyceride as an indicator of obesity were higher in NSE/hPen-2 Tg mice than in non-Tg mice. Especially, the triglyceride concentration in NSE/hPen-2 Tg mice was markedly higher (2-fold) compared with non-Tg mice. In case of a diabetes indicator, the serum glucose concentration was significantly increased in the NSE/hPen-2 Tg mice compared to the non-Tg mice (Fig. 7C). Furthermore, the insulin concentrations showed a pattern opposite to that of serum glucose concentrations. NSE/hPen-2 Tg mice showed a significant decrease in insulin concentration, while that of non-Tg mice as maintained at $10.54 \mathrm{ng} / \mathrm{ml}$ (Fig. 7D). Furthermore, to investigate the effect of the alteration of glucose concentration induced by feeding behavior on Glut-1 and Glut-3 expression, the Glut-1 
A

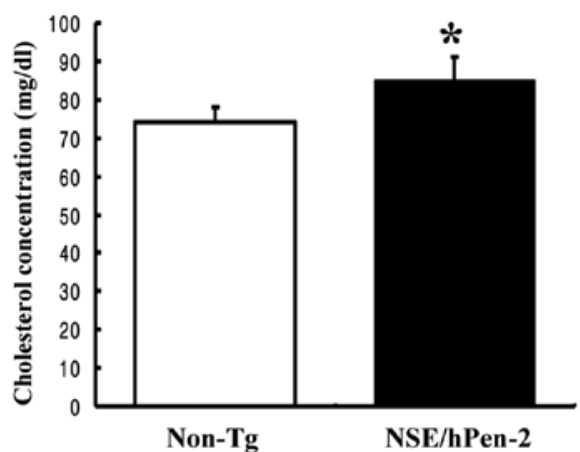

C

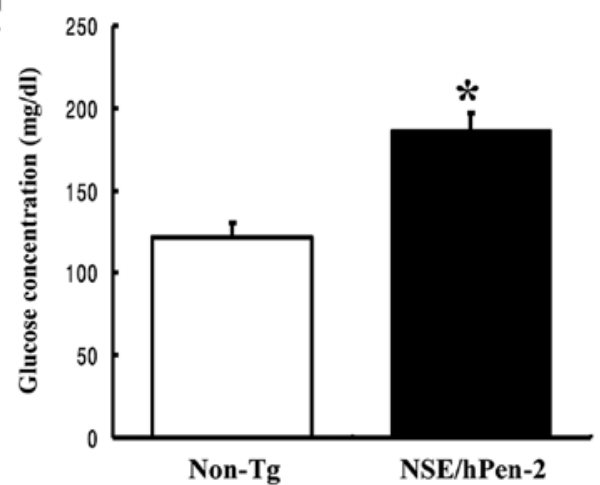

$\mathbf{E}$

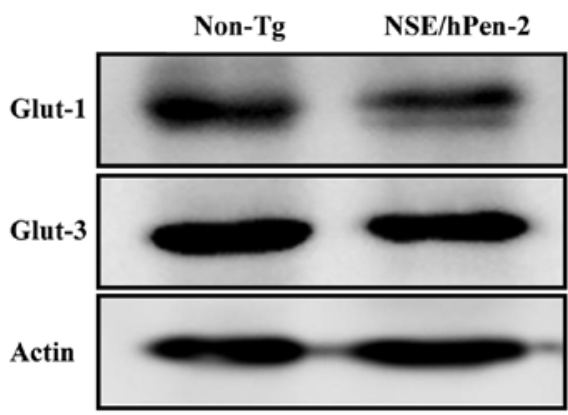

B

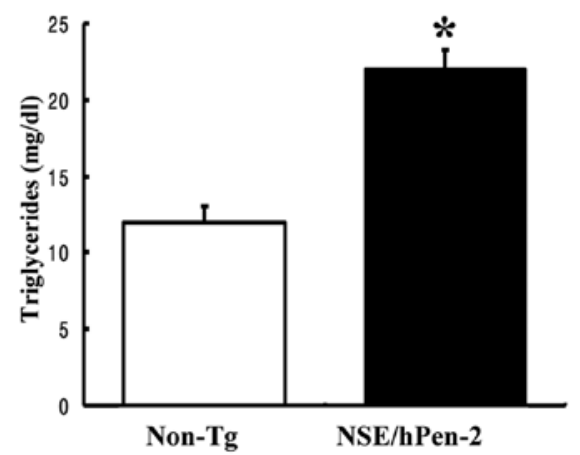

D

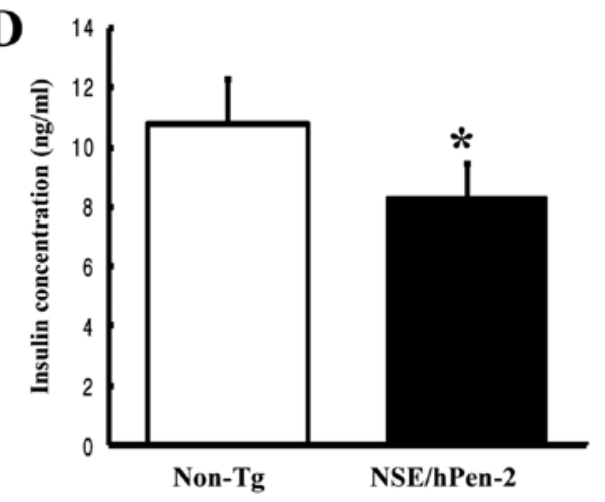

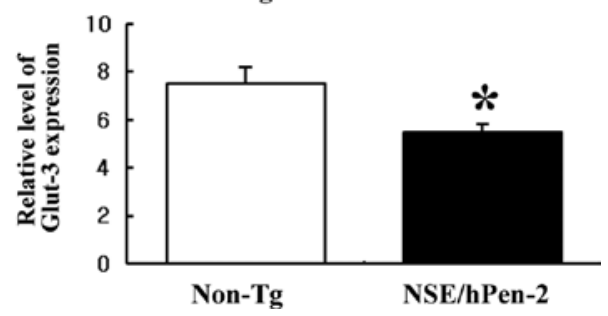

Figure 7. Alterations of indicators for diabetes and obesity, and glucose transporter expression. (A-D) Blood was collected from the abdominal vein of the mice, and serum contents of cholesterol, triglyceride and glucose were analyzed using serum biochemical analyzer. Insulin concentration was determined with an ELISA kit containing insulin specific antibody. Three experiments were assayed in triplicate using serum biochemical analysis and ELISA. (E) Glut-1 and Glut-3 protein expression in the brain were detected with anti-Glut-1 and Glut-3 primary antibody and HRP-conjugated goat anti-rabbit IgG. The densitometric intensity of the Glut-1 and Glut-3 proteins was calculated. The values represent the mean \pm SD. * P<0.05; significant difference between NSE/hPen- 2 Tg and non-Tg mice.

and Glut-3 membrane content in the brain were measured. Glut-1 and Glut-3 expression in NSE/hPen-2 Tg mice were significantly decreased by $30-40 \%$ compared to non-Tg mice (Fig. 7E). Taken together, these results indicate that the alteration of feeding behavior induced by Pen-2 overexpression may induce the key symptoms of diabetes and obesity.

\section{Discussion}

To investigate the gene function and screen for novel drugs, various $\mathrm{Tg}$ mice models were developed by microinjection of one gene of $\gamma$-secretase components under the appropriate promoter into a fertilized egg. These mice showed the AD-like phenotypes including $A \beta-42$ production, behavioral defects and an increase of $\gamma$-secretase activity. PS1 and PS2 mutant $\mathrm{Tg}$ mice showed a range of pathological and physiological alterations that mimic many aspects of $\mathrm{AD}$ and merit its more thorough examination as a model of AD $(25,26)$. Also, in a study using APH-1 knock-out mice, the deletion of APH-1induced the reduction of $\gamma$-secretase complex formation and $\beta$-amyloid secretion $(27,28)$. Nicastrin (NCT) conditional knockout mice show a progressive impairment in learning and memory, and display age-dependent cortical neuronal loss $(29,30)$. However, no studies have developed and characterized the novel Tg mice overexpressing the hPen-2 gene under the control of the NSE promoter. Presently, we developed novel 
Tg mice showing $A \beta-42$ deposition and behavioral defects. Specifically, our study explored novel characteristics, such as motor activity enhancement, feeding behavior dysfunction and the induction of diabetes in NSE/hPen-2 Tg mice. The present data are the first to indicate that hPen-2 could induce the AD-like phenotype in vivo.

Recently, the alteration of the feeding behavior in an $\mathrm{AD}$ model animal was reported for the first time $(31,32)$. APP $23 \mathrm{Tg}$ mice overexpressing human APP cDNA encoding the Swedish double mutation (K670N/M671L) under the control of the neuron-specific murine Thy-1.2 promoter showed the pathological features, learning and memory deficits analogous to AD patients (33). Especially, in a feeding behavior study using APP23 Tg mice, the mice drank more and took more pellets compared with non-Tg mice, although the olfactory function was normal (34). In the present study, the food intake of NSE/ hPen-2 Tg mice was significantly increased, similar to the results reported in APP23 Tg mice. However, the water intake was strikingly different between NSE/hPen-2 Tg mice and APP23 Tg mice. The NSE/hPen-2 Tg mice developed herein drunk less water than non-Tg mice. Furthermore, the data showed an alteration of the ratio of food and water consumption in the light vs. dark phase using the food intake/water intake/motor activity monitoring system. The results suggest that the dysfunction of feeding behavior in NSE/hPen- $2 \mathrm{Tg}$ mice was more affected in the light than the dark phase.

In humans, alteration in feeding behavior is common in dementia and helps distinguish between the different types of dementia. Several reports compared and analyzed the difference in dietary and eating behavior among patients with neurodegenerative disorders such as $\mathrm{AD}$, early frontotemporal dementia (FTD) and dementia with Lewy bodies (DLB). The change in the eating behavior was significantly more common in the FTD patients than in the AD patients $(22,23)$. Also, DLB patients showed remarkable high scores than $\mathrm{AD}$ patients for eating and swallowing problems (35). However, dysfunction of the feeding behavior in $\mathrm{AD} \mathrm{Tg}$ mice has not been hitherto reported. Our results showed the possibility that the AD-like phenotypes induced by Pen-2 overexpression may regulate the feeding behavior in NSE/hPen-2 Tg mice. Studies on the correlation between feeding behavior and dementia in humans may benefit from the present findings.

A number of studies suggested that the pathogenesis of $A D$ is highly correlated with diabetes and obesity. After induced onset diabetes by administration of streptozotocin (STZ), pR5 Tg mice expressing P301L mutant Tau showed hyperphosphorylation of Tau protein in the brain (36). Also, APP/PS1 double $\mathrm{Tg}$ mice displayed increased severity in $\mathrm{AD}$ pathology involving $A \beta$ generation, neuritic plaque formation and spatial memory deficits $(37,38)$. These reports have suggested that experimental diabetes could exacerbate the pathology of $\mathrm{AD}$ in various animal models. Meanwhile, the mouse model of the combined $\mathrm{AD}$ phenotype and diabetes phenotype was used to investigate the pathological interaction between these diseases. In a study using APP23-ob/ob double mutant mice, the onset of diabetes exacerbated AD-like cognitive dysfunction, without A $\beta$ peptide deposition (39). Also, mice overexpressing APP and displaying type 1 diabetes showed a decrease of insulin receptor activity, increase of GSK3 $\beta$ activity, increase of Tau phosphorylation and $\mathrm{A} \beta$ plaque number (40). In the present study, the increase of pathological features of diabetes and obesity were detected in the NSE/hPen-2 Tg mice compared with non- $\mathrm{Tg}$ mice. These results suggest that the pathological features of $\mathrm{AD}$ induced by overexpression of hPen- 2 could exaggerate diabetes and obesity in NSE/hPen-2 Tg mice. Also, our study may provide other evidence of the pathophysiological correlation between diabetes and AD.

All of the afore-mentioned results support the suggestion that Pen-2 in NSE/hPen-2 Tg mice may play an important role in developing the pathological characteristics of AD. Therefore, NSE/hPen-2 Tg mice can serve as an animal model for AD to understand the basal mechanism of AD pathology and screen novel therapeutic drugs.

\section{Acknowledgements}

This research was supported by Bio-Scientific Research Grants funded by the Pusan National University (PNU, Bio-Scientific Research Grant) (PNU-2010-101-236).

\section{References}

1. Kimberly WT, LaVoie MJ, Ostaszewski BL, Ye W, Wolfe MS and Selkoe DJ: Gamma-secretase is a membrane protein complex comprised of presenilin, nicastrin, Aph-1, and PEN-2. Proc Natl Acad Sci USA 100: 6382-6387, 2003.

2. Francis R, McGrath G, Zhang J, Ruddy DA, Sym M, Apfeld J, Nicoll M, Maxwell M, Hai B, Ellis MC, Parks AL, Xu W, Li J, Gurney M, Myers RL, Himes CS, Hiebsch R, Ruble C, Nye JS and Curtis D: Aph-1 and Pen-2 are required for Notch pathway signaling, gamma-secretase cleavage of $\beta A P P$, and presenilin protein accumulation. Dev Cell 3: 85-97, 2002.

3. Bergman A, Hansson EM, Pursglove SE, Farmery MR, Lannfelt L, Lendahl U, Lundkvist $J$ and Naslund J: Pen-2 is sequestered the endoplasmic reticulum and subjected to ubiquitylation and proteasome-mediated degradation in the absence of presenilin. $\mathrm{J}$ Biol Chem 279: 16744-16753, 2004.

4. Crystal AS, Morais VA, Pierson TC, Pijak DS, Carlin D, Lee VM and Doms RW: Membrane topology of gamma-secretase component Pen-2. J Biol Chem 278: 20117-20123, 2003.

5. Kim SH, Ikeuchi T, Yu C and Sisodia SS: Regulated hyperaccumulation of presenilin-1 and the 'gamma-secretase' complex. Evidence for differential intramembranous processing of transmembrane substrates. J Biol Chem 278: 33992-34002, 2003.

6. Takasugi N, Tomita T, Hayashi I, Tsuruoka M, Niimura M, Takahashi Y, Thinakaran G and Iwatsubo T: The role of presenilin cofactors in the $\gamma$-secretase complex. Nature 422: 438-441, 2003.

7. Vetrivel KS, Zhang YW, Xu H and Thinakaran G: Pathological and physiological functions of presenilins. Mol Neurodegener 1: 4,2006

8. Hasegawa H, Sanjo N, Chen F, Gu YJ, Shier C, Petit A, Kawarai T, Katayama T, Schmidt SD, Mathews PM, Schmitt-Ulms G, Fraser PE and St George-Hyslop P: Both the sequence and length of the C terminus of PEN-2 are critical for intermolecular interactions and function of presenilin complexes. J Biol Chem 279: 46455-46463, 2004.

9. Prokop S, Shirotani K, Edbauer D, Haass C and Steiner H: Requirement of PEN-2 for stabilization of the presenilin N-/Cterminal fragment heterodimer within the $\gamma$-secretase complex. J Biol Chem 279: 23255-23261, 2004.

10. Steiner H, Winkler E, Edbauer D, Prokop S, Basset G, Yamasaki A, Kostka $M$ and Haass C: PEN-2 is an integral component of thegamma-secretase complex required for coordinated expression of presenilin and nicastrin. J Biol Chem 277: 39062-39065, 2002.

11. Luo WJ, Wang H, Li H, Kim BS, Shah S, Lee HJ, Thinakaran G, Kim TW, Yu G and Xu H: PEN-2 and APH-1 coordinately regulate proteolytic processing of presenilin 1. J Biol Chem 278: 7850-7854, 2003.

12. Murphy MP, Das P, Nyborg AC, Rochette MJ, Dodson MW, Loosbrock NM, Souder TM, McLendon C, Merit SL, Piper SC, Jansen KR and Golde TE: Overexpression of nicastrin increase A $\beta$ production. FASEB J 17: 1138-1140, 2003. 
13. Hu Y, Ye Y and Fortini ME: Nicastrin is required for $\gamma$-secretase cleavage of the Drosophila Notch receptor. Dev Cell 2: 69-78, 2002.

14. Dunys J, Sevalle J, Giaime E, Pardossi-Piquard R, Vitek MP, Renbaum P, Levy-Lahad E, Zhang YW, Xu H, Checler F and da Costa CA: p53-dependent control of transactivation of the Pen2 promoter by presenilins. J Cell Sci 122: 4003-4008, 2009.

15. Crystal AS, Morais VA, Fortna RR, Carlin D, Pierson TC, Wilson CA, Lee VM and Doms RW: Presenilin modulates Pen-2 levels posttranslationally by protecting it from proteasomal degradation. Biochemistry 43: 3555-3563, 2004

16. Seo SJ, Hwang DY, Cho JS, Chae KR, Kim CK, Shim SB, Jee SW, Lee SH, Sin JS, Choi SY, Kim J and Kim YK: PEN-2 overexpression induces gamma-secretase protein and its activity with amyloid $\beta-42$ production. Neurochem Res 32: 1016-1023, 2007.

17. Hwang DY, Seo S, Kim Y, Kim C, Shim S, Jee S, Lee S, Sin J, Cho J, Kang B, Jang I and Cho J: Significant change in insulin production, glucose tolerance and ER stress signaling in transgenic mice coexpressing insulin-siRNA and human IDE. Int J Mol Med 19: 65-73, 2007.

18. Hwang DY, Chae KR, Kang TS, Hwang JH, Lim CH, Kang HK, Goo JS, Lee MR, Lim HJ, Min SH, Cho JY, Hong JT, Song CW, Paik SG, Cho JS and Kim YK: Alterations in behavior, amyloid beta-42, caspase- 3 , and Cox-2 in mutant PS2 transgenic mouse model of Alzheimer's disease. FASEB J 16: 805-813, 2002.

19. Prajapati KD, Sharma SS and Roy N: Up-regulation of albumin expression in focal ischemic rat brain. Brain Res 1327: 118-124, 2010

20. Lee KW, Im JY, Song JS, Lee SH, Lee HJ, Ha HY, Koh JY Gwag BJ, Yang SD, Paik SG and Han PL: Progressive neuronal loss and behavioral impairments of transgenic C57BL/6 inbred mice expressing the carboxy terminus of amyloid precursor protein. Neurobiol Dis 22: 10-24, 2006.

21. Fujimoto W, Shiuchi T, Miki T, Minokoshi Y, Takahashi Y, Takeuchi A, Kimura K, Saito M, Iwanaga T and Seino S: Dmbx1 is essential in agouti-related protein action. Proc Natl Acad Sci USA 104: 15514-15519, 2007.

22. Ikeda M, Brown J, Holland AJ, Fukuhara R and Hodges JR: Changes in appetite, food preference, and eating habits in frontotemporal dementia and Alzheimer's disease. J Neurol Neurosurg Psychiatry 73: 371-376, 2002.

23. Mendez MF, Licht EA and Shapira JS: Changes in dietary or eating behavior in frontotemporal dementia versus Alzheimer's disease. Am J Alzheimers Dis Other Demen 23: 280-285, 2008.

24. Whitmore C: Type 2 diabetes and obesity in adults. Br J Nurs 19 $880,882-886,2010$

25. Götz J and Ittner LM: Animal models of Alzheimer's disease and frontotemporal dementia. Nat Rev Neurosci 9: 532-544, 2008.

26. Elder GA, Gama Sosa MA, De Gasperi R, Dickstein DL and Hof PR: Presenilin transgenic mice as models of Alzheimer's disease. Brain Struct Funct 214: 127-143, 2010

27. Ma G, Li T, Price DL and Wong PC: APH-1a is the principal mammalian APH-1 isoform present in gamma-secretase complexes during embryonic development. J Neurosci 25: 192-198, 2005.
28. Serneels L, Dejaegere T, Craessaerts K, Horré K, Jorissen E, Tousseyn T, Hébert S, Coolen M, Martens G, Zwijsen A, Annaert W, Hartmann D and De Strooper B: Differential contribution of the three Aphl genes to gamma-secretase activity in vivo. Proc Natl Acad Sci USA 102: 1719-1724, 2005.

29. Nguyen V,Hawkins C, Bergeron C, Supala A, Huang J, Westaway D, St George-Hyslop P and Rozmahel R: Loss of nicastrin elicits an apoptotic phenotype in mouse embryos. Brain Res 1086: 76-84, 2006.

30. Tabuchi K, Chen G, Südhof TC and Shen J: Conditional forebrain inactivation of nicastrin causes progressive memory impairment and age-related neurodegeneration. J Neurosci 29: 7290-7301, 2009.

31. Shatenstein B, Kergoat MJ, Reid I and Chicoine ME: Dietary intervention in older adults with early-stage Alzheimer dementia: early lessons learned. J Nutr Health Aging 12: 461-469, 2008.

32. Kohjima M, Sun Y and Chan L: Increased food intake leads to obesity and insulin resistance in the tg2576 Alzheimer's disease mouse model. Endocrinology 151: 1532-1540, 2010.

33. Sturchler-Pierrat C, Abramowski D, Duke M, Wiederhold KH, Mistl C, Rothacher S, Ledermann B, Bürki K, Frey P, Paganetti PA, Waridel C, Calhoun ME, Jucker M, Probst A, Staufenbiel M and Sommer B: Two amyloid precursor protein transgenic mouse models with Alzheimer disease-like pathology. Proc Natl Acad Sci USA 94: 13287-13292, 1997.

34. Vloeberghs E, Van Dam D, Franck F, Serroyen J, Geert M, Staufenbiel M and De Deyn PP: Altered ingestive behavior, weight changes, and intact olfactory sense in an APP overexpression model. Behav Neurosci 122: 491-497, 2008.

35. Shinagawa S, Adachi H, Toyota Y, Mori T, Matsumoto I, Fukuhara R and Ikeda M: Characteristics of eating and swallowing problems in patients who have dementia with Lewy bodies. Int Psychogeriatr 21: 520-525, 2009.

36. Ke YD, Delerue F, Gladbach A, Gotz J and Ittner LM: Experimental diabetes mellitus exacerbates tau pathology in a transgenic mouse model of Alzheimer's disease. PLoS One 4: e7917, 2009.

37. Burdo JR, Chen Q, Calcutt NA and Schubert D: The pathological interaction between diabetes and presymptomatic Alzheimer's disease. Neurobiol Aging 30: 1910-1917, 2009.

38. Wang X, Zheng W, Xie JW, Wang T, Wang SL, Teng WP and Wang ZY: Insulin deficiency exacerbates cerebral amyloidosis and behavioral deficits in an Alzheimer transgenic mouse model. Mol Neurodegener 5: 46, 2010.

39. Takeda S, Sato N, Uchio-Yamada K, Sawada K, Kunieda T, Takeuchi D, Kurinami H, Shinohara M, Rakugi Hand Morishita R: Diabetes-accelerated memory dysfunction via cerebrovascular inflammation and $\mathrm{A} \beta$ deposition in an Alzheimer mouse model with diabetes. Proc Natl Acad Sci USA 107: 7036-7041, 2010.

40. Jolivalt CG, Hurford R, Lee CA, Dumaop W, Rockenstein E and Masliah E: Type 1 diabetes exaggerates features of Alzheimer's disease in APP transgenic mice. Exp Neurol 223: 422-431, 2010. 